\title{
Thyroid dysfunction in patients with childhood-onset medulloblastoma or primitive neuroectodermal tumor
}

Seung Young Jin, $M D^{1}$, Jung Yoon Choi, MD ${ }^{1,2}$, Kyung Duk Park, MD, PhD ${ }^{1,2}$, Hyoung Jin Kang, MD, PhD ${ }^{1,2}$, Hee Young Shin, MD, PhD ${ }^{1,2}$, Ji Hoon Phi, $M D, P h D^{3}$, Seung-Ki Kim, MD, $\mathrm{PhD}^{3}$, Kyu-Chang Wang, MD, $\mathrm{PhD}^{3}$, II Han Kim, $\mathrm{MD}^{4}$, Young Ah Lee, MD, PhD', Choong Ho Shin, MD, PhD', Sei Won Yang, $\mathrm{MD}, \mathrm{PhD}^{1}$

${ }^{1}$ Department of Pediatrics, Seoul National University College of Medicine, Seoul, ${ }^{2}$ Cancer Research Institute, Seoul National University College of Medicine, Seoul, ${ }^{3}$ Department of Neurosurgery, Seoul National University College of Medicine, Seoul, ${ }^{4}$ Department of Radiation Oncology, Seoul National University College of Medicine, Seoul, Korea

Received: 12 September, 2017 Revised: 16 October, 2017 Accepted: 22 October, 2017

Address for correspondence: Young Ah Lee, MD, PhD

Department of Pediatrics, Seoul National University Children's Hospital, Seoul National University College of Medicine, 101 Daehak-ro, Jongno-gu, Seoul 03080, Korea

Tel: +82-2-2072-2308

Fax: +82-2-2072-3917

E-mail: nina337@snu.ac.kr

https://orcid.org/0000-0001-91791247
Purpose: We investigated the clinical characteristics of patients who developed thyroid dysfunction and evaluated the risk factors for hypothyroidism following radiotherapy and chemotherapy in pediatric patients with medulloblastoma or primitive neuroectodermal tumor (PNET).

Methods: The medical records of 66 patients (42 males) treated for medulloblastoma $(n=56)$ or PNET $(n=10)$ in childhood between January 2000 and December 2014 at Seoul National University Children's Hospital were retrospectively reviewed. A total of 21 patients (18 high-risk medulloblastoma and 3 PNET) underwent high-dose chemotherapy and autologous stem cell rescue (HDCT/ASCR)

Results: During the median 7.6 years of follow-up, 49 patients (74\%) developed transient $(n=12)$ or permanent $(n=37)$ hypothyroidism at a median 3.8 years of follow-up (2.9-4.6 years). Younger age ( $<5$ years) at radiation exposure $(P=0.014$ vs. $\geq 9$ years $)$ and $\mathrm{HDCT}(P=0.042)$ were significantly predictive for hypothyroidism based on log-rank test. However, sex, type of tumor, and dose of craniospinal irradiation (less vs. more than $23.4 \mathrm{~Gy}$ ) were not significant predictors. Cox proportional hazard model showed that both younger age $(<5$ years $)$ at radiation exposure (hazard ratio $[\mathrm{HR}], 3.1$; vs. $\geq 9$ years; $P=0.004)$ and $\mathrm{HDCT}(\mathrm{HR}, 2.4 ; P=0.010)$ were significant predictors of hypothyroidism.

Conclusion: Three-quarters of patients with pediatric medulloblastoma or PNET showed thyroid dysfunction, and over half had permanent thyroid dysfunction. Thus, frequent monitoring of thyroid function is mandatory in all patients treated for medulloblastoma or PNET, especially, in very young patients and/or high-risk patients recommended for HDCT/ASCR.

Keywords: Thyroid hormones, Medulloblastoma, Primitive neuroectodermal tumors, Craniospinal irradiation, Chemotherapy, Adjuvant, Pediatrics

\section{Introduction}

Medulloblastoma and primitive neuroectodermal tumor (PNET) account for approximately $15 \%$, and $3 \%$ of pediatric malignant tumors of the central nervous system (CNS), respectively. ${ }^{1)}$ Treatment strategies include radical resection, followed by craniospinal irradiation (CSI), booster radiation therapy, and chemotherapy. For some high-risk patients, high-dose chemotherapy with autologous stem cell rescue (HDCT/ASCR) is indicated. ${ }^{2}$ Since survival rates markedly increased over the last 4 decades, leading to $70 \%-85 \%$ of cure rate in pediatric patients with medulloblastoma or PNET,") regular monitoring of long-term complications related to radiotherapy and chemotherapy is needed to improve quality of life.

Since thyrotoxic and hypothyroid patients following head and neck irradiation therapy were firstly reported in the 1920s, and in the 1960s, respectively, ${ }^{4}$ thyroid dysfunction after 
radiotherapy has been reported in several studies. ${ }^{5-7)}$ In addition, negative impact of chemotherapeutic agents on thyroid function has been recently reported. ${ }^{8,9)}$

We aimed to investigate clinical characteristics of pediatric patients with medulloblastoma or PNET who developed thyroid dysfunction. We also evaluated risk factors for thyroid dysfunction following radiotherapy and chemotherapy in pediatric medulloblastoma or PNET.

\section{Materials and methods}

\section{Subjects}

The retrospective reviews of pediatric patients diagnosed with medulloblastoma $(n=116)$ or PNET $(n=24)$ between January, 2000 and December, 2014 at Seoul National University Children's Hospital were performed. Although the term PNET, a heterogeneous groups of neoplasm, has been removed from the diagnostic lexicon in the World Health Organization (WHO) 2016 update classification of CNS embryonal tumors, ${ }^{10)}$ this retrospective study used PNET according to the WHO 2007 classification. All patients had undergone neurosurgery followed by radiotherapy and chemotherapy. Thyroid function was regularly followed up in only 80 patients (65 medulloblastoma and 15 PNET) in pediatric endocrine clinic ( $\geq 3$ visits). Among 65 patients with medulloblastoma, 4 hypothyroid patients before tumor diagnosis and 5 patients who received additional radiotherapy due to primary tumor relapse were excluded. Among 15 PNET patients, 2 hypothyroid patients before tumor diagnosis, 2 patients who received additional radiotherapy due to relapse and 1 patient who developed leukemia as a second malignancy were excluded. Finally, a total of 66 patients (medulloblastoma $[\mathrm{n}=56]$ and PNET $[\mathrm{n}=10]$ ) were included in this study (Fig. 1). This study was approved by the Institutional Review Board (IRB) of Seoul National University Hospital (H1708-160-880). Informed consent was waived by the IRB.

Thyroid function test were regularly followed up at interals of 3 to 6 months using immunoradiometric assay
(IRMA), radioimmunoassay (RIA) and/or chemiluminescent microparticle immune assay (CMIA). Serum concentrations of free thyroxine (fT4) and thyroid stimulating hormone (TSH) using IRMA kits (RIAKEY, Shin Jin Medics, Seoul, Korea), and serum levels of triiodothyronine (T3), using RIA kits (Brahms DYNOTest; Diagnostica GmbH, Berlin, Germany) were analyzed. The normal ranges of serum fT4, TSH, and T3 using IRMA and RIA were $0.70-1.80 \mathrm{ng} / \mathrm{dL}, 0.4-4.1 \mathrm{mIU} / \mathrm{L}$, and 87-184 ng/dL, respectively. Serum concentrations of fT4, TSH, and T3 were also measured using CMIA on an Architect i2000 system (Abbott Korea, Seoul, Korea), with the normal ranges of $0.70-1.48 \mathrm{ng} / \mathrm{dL}, 0.35-4.94 \mathrm{mIU} / \mathrm{L}$, and 58-159 $\mathrm{ng} / \mathrm{dL}$, respectively. Thyroid autoimmunity was investigated by the measurement of serum concentrations of antithyroid peroxidase antibodies (TPOAbs) and antithyroglobulin antibodies (TGAbs), using RIA kits (Brahms DYNOTest, Diagnostica GmbH, Berlin, Germany). Both titers of TPOAbs and TGAbs were considered to be positive for values higher than $60 \mathrm{U} / \mathrm{mL}$.

Pituitary function test was undertaken after final tumor treatment. Growth hormone $(\mathrm{GH})$ deficiency was diagnosed when $\mathrm{GH}$ response was inadequate (peak $\mathrm{GH}<10 \mathrm{ng} / \mathrm{mL}$ ) as a results of at least 2 stimulation tests using insulin, clonidine, or L-dopa. Adrenocorticotropic hormone (ACTH) deficiency was confirmed when peak cortisol was less than $18 \mu \mathrm{g} / \mathrm{mL}$ using insulin tolerance test or low dose ACTH stimulation test. If patient who did not present secondary sexual characteristics at pubertal age showed inadequate luteinizing hormone (LH) response (peak $\mathrm{LH}<5 \mathrm{IU} / \mathrm{L}$ ) after $\mathrm{GnRH}$ stimulation, the patient was diagnosed with gonadotropin deficiency.

Patients with medulloblastoma had received chemotherapy according to the risk stratification (average and high-risk) ${ }^{10)}$ by age at diagnosis, extent of disease, histology and response to treatment. ${ }^{11-14)}$ Twenty-three patients with average-risk medulloblastoma were treated with the "KSPNO-M051" regimen, consisting of cisplatin, cyclophosphamide, and vincristine. Thirty-three patients with high-risk medulloblastoma and 4 patients with PNET received chemotherapy consisting of cisplatin, cyclophosphamide, vincristine, etoposide,

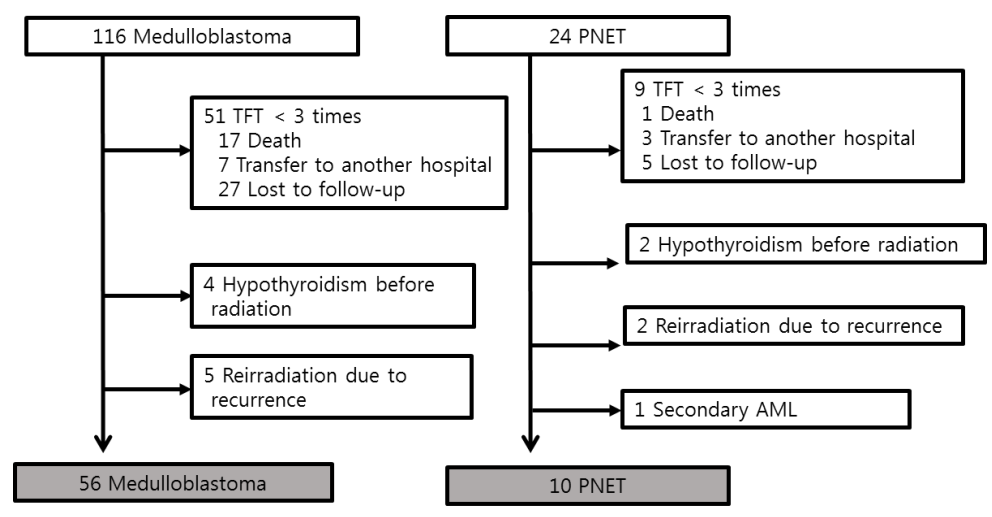

Fig. 1. Inclusion criteria of patients treated for medulloblastoma or primitive neuroectodermal tumor (PNET). TFT, thyroid function test; AML, acute myeloid leukemia. 
and ifosfamide. Other six with PNET received chemotherapy according to the protocol named eight drugs in 1 day (8 in 1 ) regimen, consisting of cisplatin, cyclophosphamide, vincristine, carmustine, procarbazine, hydroxyurea, cytarabine, and prednisone. A total of 21 patients (18 high-risk medulloblastoma and 3 PNET) underwent HDCT/ASCT. Conditioning regimens for tandem HDCT/ASCT were carboplatin-thiotepa-etoposide and cyclophosphamide-melphalan in 9 , and topotecanthiotepa-carboplatin and melphalan-etoposide-carboplatin in 5 patients, respectively. The remaining 7 patients received single HDCT/ASCT with topotecan-thiotepa-carboplatin.

\section{Statistical analysis}

The data were analyzed using IBM SPSS Statistics ver. 22.0 (IBM Co., Armonk, NY, USA). All continuous variables described as mean \pm standard deviation were compared using Student $t$-test. The chi-square or Fisher exact test was used to compare categorical variables between the 2 groups. The chi-square test for trend analysis was used to compare the proportion of variables among the three groups. Event-free survival of thyroid dysfunction was constructed using the Kaplan-Meier method, and the groups were compared by the log-rank test. Event was defined as subclinical or overt hypothyroidism. Additionally, the Cox proportional hazard model was used to find predictors for thyroid dysfunction after adjusting for covariates. The hazard ratio (HR), $95 \%$ confidence interval (CI), and $P$-value were reported. Values of $P<0.05$ were considered statistically significant.

\section{Table 1. Patient characteristics}

\begin{tabular}{|c|c|}
\hline Characteristic & Value \\
\hline Age (yr), mean $\pm S D$ & $7.1 \pm 3.4$ \\
\hline Male sex & $42(63.6)$ \\
\hline Tumor diagnosis (medulloblastoma: PNET) & $56(84.8): 10(15.2)$ \\
\hline Follow-up (yr), mean \pm SD (range) & $7.6 \pm 3.6(1.7-14.5)$ \\
\hline Craniospinal irradiation (CSI) dose (Gy) & $23.2 \pm 4.9$ \\
\hline Craniospinal irradiation (CSI) dose $<23.4$ Gy & $10(15.2)$ \\
\hline $\begin{array}{l}\text { Craniospinal irradiation (CSI) + boost (Gy), } \\
\text { mean } \pm \text { SD }\end{array}$ & $54.2 \pm 12.6$ \\
\hline $\begin{array}{l}\text { High dose chemotherapy/ } \\
\text { autologous stem cell rescue (HDCT/ASCR) }\end{array}$ & $21(31.8)$ \\
\hline Thyroid dysfunction & $49(74.2)$ \\
\hline Normal fT4, elevated TSH (5 to $10 \mathrm{mlU} / \mathrm{L}$ ) & $27(40.9)$ \\
\hline Normal fT4, elevated TSH ( $\geq 10$ mIU/L) & $9(13.6)$ \\
\hline Decreased fT4, suppressed or normal TSH & $1(1.5)$ \\
\hline Decreased fT4, elevated TSH (5 to $10 \mathrm{mIU} / \mathrm{L}$ ) & $4(6.1)$ \\
\hline Decreased fT4, elevated TSH ( $\geq 10$ mIU/L) & $8(12.1)$ \\
\hline Levothyroxine medication & $33(50)$ \\
\hline Growth hormone deficiency & $36(54.5)$ \\
\hline Growth hormone therapy & $30(45.5)$ \\
\hline Hydrocortisone medication & $10(15.2)$ \\
\hline Sex hormone therapy & $9(13.6)$ \\
\hline
\end{tabular}

Values are presented as number (\%) unless otherwise indicated.

SD, standard deviation; PNET, primitive neuroectodermal tumor; fT4, free thyroxine; TSH, thyroid stimulating hormone.

\section{Results}

\section{Patient characteristics}

Table 1 showed clinical characteristics of 66 patients (42 males). The mean age at tumor diagnosis was 7.1 years $(2.7-15.4$ years). All patients except one (2.7-year-old boy) had received CSI (23.2 \pm 4.9 Gy, 19.8-30.6 Gy). Age at radiation exposure was classified into the 3 groups as follows; $<5$ years $(\mathrm{n}=24), 5.0$ to 8.9 years $(\mathrm{n}=22)$ and $\geq 9$ years $(\mathrm{n}=20)$. Ten patients received less than 23.4 Gy of CSI and 21 high-risk patients underwent HDCT/ ASCR (Table 1).

During the median follow-up of 7.6 years (1.7-14.5 years), 49 patients (74.2\%) developed thyroid dysfunction, 33 of whom eventually took levothyroxine medication. Thyroid dysfunction was presented as follows; (1) normal fT4 and slightly elevated TSH $(<10 \mathrm{mIU} / \mathrm{L})(\mathrm{n}=27),(2)$ normal fT4, elevated TSH $(\geq 10$ $\mathrm{mIU} / \mathrm{L})(\mathrm{n}=9)$, (3) decreased fT4 and decreased or normal TSH $(n=1),(4)$ decreased fT4 and slightly elevated TSH $(<10$ $\mathrm{mIU})(\mathrm{n}=4)$, and $(5)$ decreased fT4, elevated TSH $(\geq 10 \mathrm{mIU} / \mathrm{L})$ $(\mathrm{n}=8)$. During follow-up, none of 24 patients who underwent thyroid autoantibody evaluations showed positive thyroid autoimmunity. All patients underwent pituitary function testing after the final tumor treatment. Thirty-six patients (54.5\%) were diagnosed with GH deficiency, 30 of whom were treated with GH therapy. Ten patients (15.2\%) received hydrocortisone and 9 patients (13.6\%) received sex hormone replacement therapy (Table 1). No significant difference in proportion of patients with GH, ACTH, and gonadotropin deficiency between patients with thyroid dysfunction or those without.

\section{Characteristics of patients developing thyroid dysfunction}

Patients were categorized into the 3 groups according to thyroid function. If free T4 was decreased and/or TSH was elevated, patients were categorized into thyroid dysfunction group $(n=49)$. If thyroid dysfunction was normalized without levothyroxine medication $(\mathrm{n}=10)$ or after discontinuation of levothyroxine medication $(n=2)$, it was transient. If patients showed persistent subclinical hypothyroidism $(\mathrm{n}=6)$ or required persistent levothyroxine medication $(\mathrm{n}=31)$, they belonged to persistent group. As a result, thyroid dysfunction group was classified into normal $(\mathrm{n}=17)$, and transient thyroid dysfunction $(n=12)$ and persistent thyroid dysfunction $(n=37)$ groups. Table 2 showed comparison of clinical characteristics among the 3 groups.

Younger age at radiation exposure was significantly associated with increased risk for permanent thyroid dysfunction $(P<0.001$ by chi-square test for trend analysis) (Table 2). There were no significant sex and tumor differences in the risk for thyroid dysfunction among the three groups. The proportion of patients developing thyroid dysfunction was not significantly different according to whether patients received less than $23.4 \mathrm{~Gy}$ of CSI or more. Patients who received HDCT/ASCR had a higher risk 
for thyroid dysfunction with marginal significance $(P=0.079)$ (Table 2).

\section{Predictors for thyroid dysfunction in pediatric patients with Medulloblastoma or PNET}

We used the Kaplan-Meier method to construct event (hypothyroidism)-free survival curves. Time to period to thyroid dysfunction was a median 3.8 years (95\% CI, 2.9-4.6) (Fig. 2A). Younger age ( $<5$ years) at radiation exposure was significantly predictive for thyroid dysfunction ( $P=0.014$ vs. $\geq 9$ years) (Fig. 2B). Sex, type of tumor, and dose of CSI (less vs. more than 23.4 Gy) were not significant predictors. However, event-free survival of patients who received HDCT was significantly lower than those who did not $(P=0.042)$ (Fig. 2C). Finally, Cox proportional hazards model showed that both younger age ( $<5$ years) at the time of radiation therapy (HR, 3.1; vs. $\geq 9$ years; $P=0.004)$ and $\operatorname{HDCT}(\mathrm{HR}, 2.4 ; P=0.010)$ were significant predictors for thyroid dysfunction in those with medulloblastoma or PNET (Table 3).

\section{Discussion}

During the median 7.6 years of follow-up, three-quarters (74\%) of patients with childhood-onset medulloblastoma or PNET showed thyroid dysfunction (18\% transient and 56\% permanent) within the first 5 years of treatment. Younger age at the time of radiotherapy ( $<5$ years of age) and HDCT for highrisk patients were significantly predictive for developing thyroid dysfunction.

Endocrine toxicity due to radiation damage to the hypothalamus, pituitary and thyroid has been documented in childhood brain tumor survivors. ${ }^{15-17)}$ Hypothyroidism is one of early endocrine disorders after CSI. ${ }^{7,15}$ Hypothyroidism in survivors of childhood brain tumors resulted from either direct irradiation of the thyroid gland (primary hypothyroidism) and/ or irradiation of the hypothalamic-pituitary gland complex (central hypothyroidism). Among childhood cancer survivors whose brain tumor did not directly invade hypothalamus and pituitary, thyroid dysfunction, mostly hypothyroidism, was reported in up to $60 \%$ following radiotherapy usually within the

Table 2. Characteristics of patients developing thyroid dysfunction

\begin{tabular}{|c|c|c|c|c|}
\hline \multirow{2}{*}{ Variable } & \multirow{2}{*}{ Normal } & \multicolumn{2}{|c|}{ Thyroid dysfunction } & \multirow{2}{*}{$P$-value ${ }^{\dagger}$} \\
\hline & & Transient & Permanent & \\
\hline No of patients & $17(25.7)$ & $12(18.2)$ & $37(56.1)$ & \\
\hline Male:female, n (male\%) & $11: 6(64.7)$ & $7: 5(58.4)$ & 24: $13(64.9)$ & 0.935 \\
\hline Age at radiation therapy, $<5: 5-8.9: \geq 9$ years & $1: 6: 10(5.9: 35.3: 58.8)$ & 5:3:4 (41.7:25: 3.3) & 18: 3:6 (48.6:35.1:16.3) & $0.001^{*}$ \\
\hline Medulloblastoma: PNET & $16: 1(94.1: 5.9)$ & $11: 1(91.7: 8.3)$ & $29: 8(78.4: 21.6)$ & 0.113 \\
\hline CSI dose, $<23.4$ Gy & $1(5.9)$ & $1(8.3)$ & $8(21.6)$ & 0.250 \\
\hline HDCT/ASCT, yes & $5(25)$ & $0(0)$ & $16(76.2)$ & 0.079 \\
\hline
\end{tabular}

Values are presented as number (\%) unless otherwise indicated.

PNET, primitive neuroectodermal tumor; CSI, craniospinal irradiation; HDCT, high-dose chemotherapy; ASCR, autologous stem cell rescue ${ }^{*} \mathrm{P}<0.05$, significant difference. ${ }^{\dagger} \mathrm{Chi}-$ square test for trend analysis.
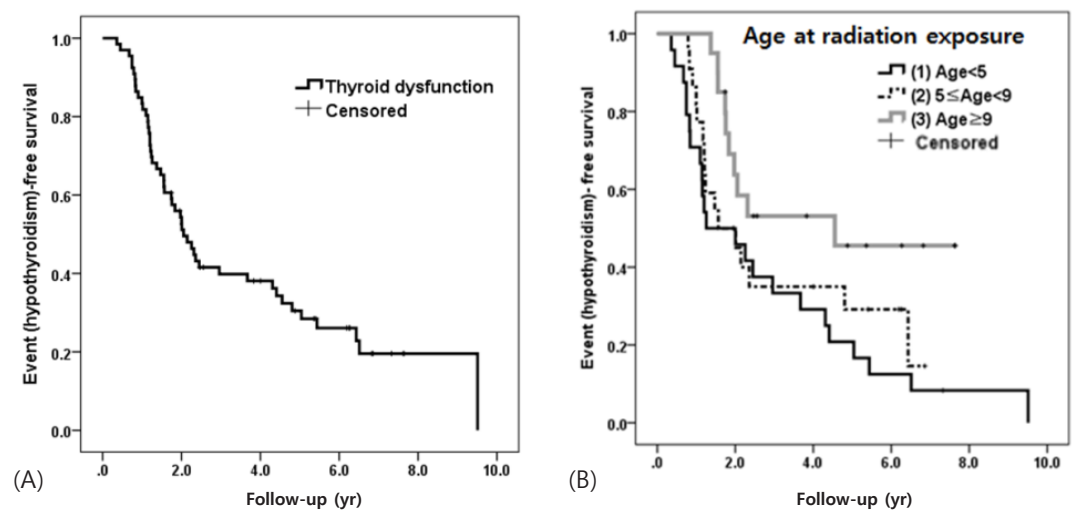

\begin{tabular}{lcc}
\hline Age $(\mathrm{yr})$ & HR $(95 \%$ C.I $)$ & $P$-value \\
\hline$\geq 9$ & Reference & \\
$<5$ & $2.6(1.2-5.4)$ & 0.014 \\
$5.0-8.9$ & $1.9(0.88-4.3)$ & 0.102 \\
\hline
\end{tabular}

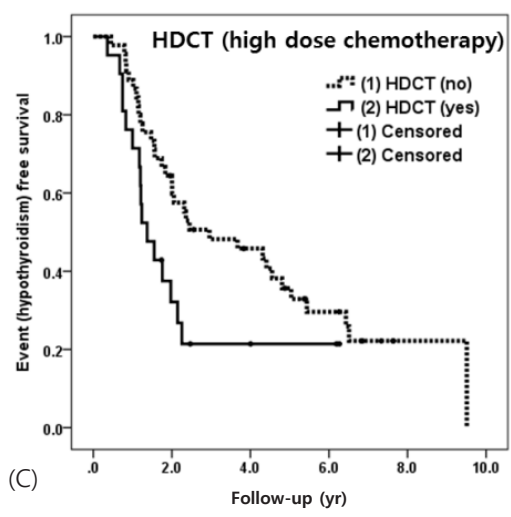

\begin{tabular}{lcc}
\hline HDCT & HR $(95 \%$ C.I $)$ & $P$-value \\
\hline No & Reference & \\
Yes & $1.9(1.01-3.5)$ & 0.042 \\
\hline
\end{tabular}

Fig. 2. (A) Time to period to thyroid dysfunction was a median 3.8 years ( $95 \%$ confidence interval [Cl], 2.9-4.6). Younger age (<5 years) at radiation exposure (B) and highdose chemotherapy $(\mathrm{HDCT})(\mathrm{C})$ and autologous stem cell rescue were predictors for the development of hypothyroidism during follow-up. HR, hazard ratio. 
first 5 years of treatment. ${ }^{7,17-20)}$ As shown in Table 4, more than half of patients with medulloblastoma and/or PNET showed thyroid dysfunction as follow-up duration increased to 8 years, and primary hypothyroidism is more prevalent than central hypothyroidism. ${ }^{7,18)}$ In this study, three-quarters of patients developed subclinical or overt hypothyroidism at a median time of 3.8 years (2.9-4.6 years). Although the timing of starting medication was different between three endocrinologists, more than half of patients were at significant risk for permanent hypothyroidism requiring levothyroxine in our study. And it is mandatory to monitor the functions of the thyroid gland in children treated for medulloblastoma or PNET.

This study included homogenous subjects treated for medulloblastoma or PNET. Although previous studies did not show consistent results, ${ }^{7,17)}$ younger age at radiation exposure was significantly associated with hypothyroidism in this study. Due to the devastating late effects related to head and neck irradiation in young children with brain tumors, treatment for very young patient needs to be evolved to avoid or postpone irradiation. Then, we evaluated whether low-dose CSI $(<23.4$ Gy) has a lower risk for hypothyroidism than standard dose radiotherapy ( $\geq 23.4 \mathrm{~Gy})$. As a result, there was no difference in risk for hypothyroidism according to the dose of CSI (less vs. more than 23.4 Gy) in this study, in consistent with previous report. ${ }^{7)}$ According to recent study on Korean childhood cancer survivors from brain and nasopharyngeal tumor, Hodgkin's lymphoma or leukemia, ${ }^{6}$ head irradiation dose $\geq 18$ Gy was a significant risk factor for persistent subclinical hypothyroidism. Since embryonal CNS tumor patients who underwent similar

Table 3. Predictors for developing thyroid dysfunction in patients with medulloblastoma or PNET

\begin{tabular}{lccccc}
\hline \multirow{2}{*}{ Variable } & \multicolumn{2}{c}{ Unadjusted } & & \multicolumn{2}{c}{ Adjusted } \\
\cline { 2 - 3 } \cline { 5 - 6 } & $H R(95 \% \mathrm{Cl})$ & $P$-value & & $H R(95 \% \mathrm{Cl})$ & $P$-value \\
\hline Age at radiation (yr) & & & & \\
$<5$ vs. $\geq 9$ & $2.6(1.2-5.4)$ & 0.014 & & $3.1(1.4-6.7)$ & 0.004 \\
$5-8.9$ vs. $\geq 9$ & $1.9(0.88-4.3)$ & 0.102 & & $1.9(0.9-4.2)$ & 0.108 \\
Male vs. female & $1.1(0.59-1.9)$ & 0.845 & & - & - \\
$\begin{array}{l}\text { Medulloblastoma } \\
\text { vs. PNET }\end{array}$ & $1.5(0.73-3.1)$ & 0.275 & & - & - \\
CSI dose $\geq 23.4$ Gy & $0.95(0.44-2.0)$ & 0.895 & & - \\
HDCT/ASCT & $2.0(1.02-3.5)$ & 0.042 & $2.4(1.2-4.5)$ & 0.010 \\
\hline HR, hazard ratio; CI, confidence interval; PNET, primitive \\
neuroectodermal tumor; CSI, craniospinal irradiation; HDCT, high-dose \\
chemotherapy; ASCR, autologous stem cell rescue.
\end{tabular}

doses of CSI (range, 19.8-30.6 Gy) for medulloblastoma or PNET were included in this study, CSI dose was not significantly predictive. Rather, age and HDCT were significant predictors for hypothyroidism in this study. Since the report of 134 survivors from childhood brain tumors not involving the hypothalamicpituitary axis showed that thyroid dysfunction was more common in children who received combined radiotherapy and chemotherapy than in those who received radiotherapy alone, ${ }^{21)}$ chemotherapeutic drugs has been suspected as candidates adversely affecting thyroid function. ${ }^{9}$ Although lomustine, vincristine, and cisplatin have shown in vitro adverse effects on thyroid cells. ${ }^{22)}$ very few studies evaluated the effects of multidrug chemotherapy on thyroid function in cancer patients, ${ }^{23,24)}$ and it still remains controversial. Although all patients received multidrug chemotherapy including vincristine and cisplatin in our study, those who received HDCT/ASCR showed higher risk for hypothyroidism. The mechanism remains unclear and it needs to be elucidated.

This retrospective study had several limitations. Due to lack of information of family history of thyroid disease and regular investigation of the thyroid autoantibodies, thyroid autoimmunity and family history of thyroid disease were not included as covariates in our analysis. In addition, since thyrotropin releasing hormone stimulation was not performed in all patients, we could not distinguish secondary (pituitary disorder) from tertiary (hypothalamic disorder) hypothyroidism.

One previous study investigated the way to prevent thyroid dysfunction ${ }^{25)}$ suggesting that TSH suppression with levothyroxine from 14 days before and up to the end of CSI had a protective effect on thyroid function at long-term followup. Further studies are needed to determine how to minimize treatment-related endocrine complications. Moreover, the incidence and risk factors for thyroid cancer as secondary malignancy need to be investigated in further studies.

In conclusion, thyroid dysfunction was the most common endocrine complication seen in three-quarters of patients treated with medulloblastoma or PNET within the first 5 years after treatment. Younger age at radiation exposure $(<5$ years of age) and HDCT were significantly predictive for hypothyroidism. Thus, frequent monitoring of thyroid function is mandatory in all patients treated for medulloblastoma or PNET, especially, in very young patients and/or high-risk patients indicated for HDCT/ASCR.

Table 4. Previous studies to evaluate thyroid dysfunction in patients with medulloblastoma and/or PNET

\begin{tabular}{|c|c|c|c|c|c|c|}
\hline Study & Tumor diagnosis & $\begin{array}{c}\text { No. of } \\
\text { patients }\end{array}$ & Boys, n (\%) & $\begin{array}{l}\text { Mean age at } \\
\text { radiation therapy (yr) }\end{array}$ & $\begin{array}{l}\text { Mean follow-up } \\
\text { duration (yr) }\end{array}$ & No. of hypothyroid patients, $n$ (\%) \\
\hline Sobol et al. $(2012)^{20)}$ & Medulloblastoma & 23 & $11(47.8)$ & 9.5 & Not available & 12 (52.1): Hypothyroidism \\
\hline Chin et al. $(1997)^{18)}$ & Medulloblastoma/PNET & 36 & $20(55.6)$ & 8.4 & 8.3 & $\begin{array}{l}21 \text { (58.3): Primary } \\
2 \text { (5.5\%): Central }\end{array}$ \\
\hline Paulino $(2002)^{7)}$ & Medulloblastoma & 32 & $22(68.7)$ & 7.2 & 8.2 & $\begin{array}{l}12 \text { (37.5): Primary } \\
6 \text { (18.8): Central }\end{array}$ \\
\hline Yoon et al. $(2006)^{19)}$ & Medulloblastoma & 37 & $24(64.9)$ & 7.2 & 3.3 & 6 (16.2\%): Hypothyroidism \\
\hline Our study & Medulloblastoma & 66 & $42(63.6)$ & 7.1 & 7.6 & 49 (74.2\%) : Hypothyroidism \\
\hline
\end{tabular}




\section{Conflict of interest}

No potential conflict of interest relevant to this article was reported.

\section{References}

1. Ostrom QT, Gittleman H, Xu J, Kromer C, Wolinsky Y, Kruchko C, et al. CBTRUS statistical report: primary brain and other central nervous system tumors diagnosed in the United States in 2009-2013. Neuro Oncol 2016;18(suppl_5):v1-75.

2. Massimino M, Biassoni V, Gandola L, Garrè ML, Gatta G, Giangaspero F, et al. Childhood medulloblastoma. Crit Rev Oncol Hematol 2016;105:35-51.

3. Packer RJ, Gajjar A, Vezina G, Rorke-Adams L, Burger PC, Robertson PL, et al. Phase III study of craniospinal radiation therapy followed by adjuvant chemotherapy for newly diagnosed average-risk medulloblastoma. J Clin Oncol 2006;24:4202-8.

4. Jereczek-Fossa BA, Alterio D, Jassem J, Gibelli B, Tradati N, Orecchia R. Radiotherapy-induced thyroid disorders. Cancer Treat Rev 2004;30:369-84.

5. Constine LS, Donaldson SS, McDougall IR, Cox RS, Link MP, Kaplan HS. Thyroid dysfunction after radiotherapy in children with Hodgkin's disease. Cancer 1984;53:878-83.

6. Lee HJ, Hahn SM, Jin SL, Shin YJ, Kim SH, Lee YS, et al. Subclinical hypothyroidism in childhood cancer survivors. Yonsei Med J 2016;57:915-22.

7. Paulino AC. Hypothyroidism in children with medulloblastoma: a comparison of 3600 and 2340 cGy craniospinal radiotherapy. Int J Radiat Oncol Biol Phys 2002;53:543-7.

8. Hamnvik OP, Larsen PR, Marqusee E. Thyroid dysfunction from antineoplastic agents. J Natl Cancer Inst 2011;103: 1572-87.

9. Yeung SC, Chiu AC, Vassilopoulou-Sellin R, Gagel RF. The endocrine effects of nonhormonal antineoplastic therapy. Endocr Rev 1998;19:144-72.

10. Sarkar C, Deb P, Sharma MC. Medulloblastomas: new directions in risk stratification. Neurol India 2006;54:16-23.

11. Kim H, Kang HJ, Lee JW, Park JD, Park KD, Shin HY, et al. Irinotecan, vincristine, cisplatin, cyclophosphamide, and etoposide for refractory or relapsed edulloblastoma/PNET in pediatric patients. Childs Nerv Syst 2013;29:1851-8.

12. Park JE, Kang J, Yoo KH, Sung KW, Koo HH, Lim DH, et al. Efficacy of high-dose chemotherapy and autologous stem cell transplantation in patients with relapsed medulloblastoma: a report on the Korean Society for Pediatric Neuro-Oncology (KSPNO)-S-053 study. J Korean Med Sci 2010;25:1160-6.

13. Shih CS, Hale GA, Gronewold L, Tong X, Laningham FH,
Gilger EA, et al. High-dose chemotherapy with autologous stem cell rescue for children with recurrent malignant brain tumors. Cancer 2008;112:1345-53.

14. Yoon JH, Park KD, Kang HJ, Kim H, Lee JW, Kim SK, et al. Treatment of pediatric average-risk medulloblastoma using craniospinal irradiation less than $2500 \mathrm{cGy}$ and chemotherapy: single center experience in Korea. World J Pediatr 2017;13:367-73.

15. Clement SC, Schouten-van Meeteren AY, Boot AM, Claahsen-van der Grinten HL, Granzen B, Sen Han K, et al. Prevalence and risk factors of early endocrine disorders in childhood brain tumor survivors: a nationwide, multicenter study. J Clin Oncol 2016;34:4362-70.

16. Rose SR, Horne VE, Howell J, Lawson SA, Rutter MM, Trotman GE, et al. Late endocrine effects of childhood cancer. Nat Rev Endocrinol 2016;12:319-36.

17. Saha A, Salley CG, Saigal P, Rolnitzky L, Goldberg J, Scott $\mathrm{S}$, et al. Late effects in survivors of childhood CNS tumors treated on Head Start I and II protocols. Pediatr Blood Cancer 2014;61:1644-52.

18. Chin D, Sklar C, Donahue B, Uli N, Geneiser N, Allen J, et al. Thyroid dysfunction as a late effect in survivors of pediatric medulloblastoma/primitive neuroectodermal tumors: a comparison of hyperfractionated versus conventional radiotherapy. Cancer 1997;80:798-804.

19. Yoon IS, Seo JY, Shin CH, Kim IH, Shin HY, Yang SW, Ahn HS. Endocrine dysfunction and growth in children with medulloblastoma. Korean Journal of Pediatrics. 2006;49:292-7.

20. Sobol G, Musioł K, Kalina M, Kalina-Faska B, MiziaMalarz A, Ficek K, et al. The evaluation of function and the ultrasonographic picture of thyroid in children treated for medulloblastoma. Childs Nerv Syst 2012;28:399-404.

21. Ogilvy-Stuart AL, Shalet SM, Gattamaneni HR. Thyroid function after treatment of brain tumors in children. J Pediatr 1991;119:733-7.

22. Massart C, Le Tellier C, Lucas C, Gibassier J, Leclech G, Nicol M. Effects of cisplatin on human thyrocytes in monolayer or follicle culture. J Mol Endocrinol 1992;8:2438.

23. Stuart NS, Woodroffe CM, Grundy R, Cullen MH. Longterm toxicity of chemotherapy for testicular cancer--the cost of cure. Br J Cancer 1990;61:479-84.

24. Torino F, Barnabei A, Paragliola R, Baldelli R, Appetecchia M, Corsello SM. Thyroid dysfunction as an unintended side effect of anticancer drugs. Thyroid 2013;23:1345-66.

25. Massimino M, Gandola L, Collini P, Seregni E, Marchianò A, Serra A, et al. Thyroid-stimulating hormone suppression for protection against hypothyroidism due to craniospinal irradiation for childhood medulloblastoma/primitive neuroectodermal tumor. Int J Radiat Oncol Biol Phys 2007;69:404-10. 